\section{Gut microbiota changes are detected in asymptomatic very young children with SARS- CoV-2 infection}

We read with great interest the recent article by Yeoh et al, demonstrating an altered stool microbiome composition in patients with COVID-19 compared with controls, with greater dysbiosis correlating with elevated inflammatory markers. ${ }^{1}$ Additionally, dysbiosis was seen after disease resolution. ${ }^{1}$

To our knowledge, gut microbiome studies in young children with COVID-19 have not been reported. Critically, the developing gut microbiome of very young children differs from adults and establishes immune and inflammatory pathways. ${ }^{2} 3$ Moreover, children with COVID-19 can subsequently develop autoimmune and autoinflammatory diseases including Multisystem Inflammatory Syndrome in Children (MIS-C) ${ }^{45}$, which may in part be microbiome mediated, given recent findings by Yeoh et al. ${ }^{1}$ It is difficult to study this in young children, as many with SARS-CoV-2 infection are asymptomatic and rarely tested. ${ }^{6}$

To address this, knowing that SARSCoV-2 can be detected in stool, ${ }^{7}$ we used an established study collecting longitudinal stool samples from before and throughout the pandemic to investigate the prevalence and associated microbiome changes of SARS-CoV-2 in very young children. We ran the CDC 2019Novel Coronavirus Real-Time RT-PCR Diagnostic Panel assay on 769 serial stool samples from 595 children aged 0-24 months collected from February 2020 to February 2021. The prevalence of SARS-CoV-2 in faeces was 1.7\% (13 samples from 13 separate children) with prevalence at $<2$ days and 2,6 , 12 and 24 months of $0 \%(0 / 1), 0 \%$ $(0 / 21), 2.6 \%(4 / 156), 2.0 \%$ (7/357) and $0.9 \%,(2 / 234)$, respectively. Prevalence by month is shown in online supplemental figure $1 \mathrm{~A}$, with the first positive sample detected 31 days before the first reported case of COVID-19 regionally. No samples were positive in controls collected prior to the pandemic in 2019 ( $\mathrm{n}=97$ samples from 66 individuals). Of 13 positive children, 12 were asymptomatic with no personal or family history of SARS-CoV-2 (table 1A). Of 13 children, 1 was symptomatic with COVID-19 diagnosed 21 days before stool was collected. Hispanic ethnicity

Table 1 (A) Characteristics of overall cohort and (B) characteristics of matched cohort for microbiome analysis

\begin{tabular}{|c|c|c|c|}
\hline (A) Characteristics of overall cohort & Negative $(n=582)$ & Positive $(n=13)$ & P Value \\
\hline $\begin{array}{l}\text { Hispanic Ethnicity } \\
\text { Missing=26 }\end{array}$ & $130(23.4 \%)$ & $8(61.5 \%)$ & 0.006 \\
\hline $\begin{array}{l}\text { Household member diagnosed with SARS-CoV- } 2 \\
\text { Missing }=51\end{array}$ & $19(3.6 \%)$ & $1(7.7 \%)$ & 0.4 \\
\hline $\begin{array}{l}\text { Childcare outside of the home during quarantine } \\
\text { Missing }=63\end{array}$ & $104(20.0 \%)$ & $2(15.4 \%)$ & 0.7 \\
\hline $\begin{array}{l}\text { Household with essential worker during quarantine } \\
\text { Missing }=58\end{array}$ & $269(51.3 \%)$ & $9(69.2 \%)$ & 0.2 \\
\hline (B) Characteristics of matched cohort for microbiome analysis & Negative $(n=26)$ & Positive $(n=13)$ & $P$ value \\
\hline \multicolumn{4}{|l|}{ Sex } \\
\hline Male & $12(46.2 \%)$ & $6(46.2 \%)$ & \multirow[t]{2}{*}{1} \\
\hline Female & $14(53.8 \%)$ & $7(53.8 \%)$ & \\
\hline \multicolumn{4}{|l|}{ Delivery Mode } \\
\hline Caesarean Section & $12(46.2 \%)$ & $6(46.2 \%)$ & \multirow[t]{2}{*}{1} \\
\hline Vaginal Delivery & $14(53.8 \%)$ & $7(53.8 \%)$ & \\
\hline \multicolumn{4}{|l|}{ Ethnicity } \\
\hline Hispanic & $16(61.5 \%)$ & $8(61.5 \%)$ & \multirow[t]{2}{*}{1} \\
\hline Non-Hispanic & $10(38.5 \%)$ & $5(38.5 \%)$ & \\
\hline \multicolumn{4}{|l|}{ Age at time of sample: } \\
\hline 6 months & $8(30.8 \%)$ & $4(30.8 \%)$ & \multirow[t]{3}{*}{1} \\
\hline 12 months & $14(53.8 \%)$ & $7(53.8 \%)$ & \\
\hline 24 months & $4(15.4 \%)$ & $2(15.4 \%)$ & \\
\hline \multicolumn{4}{|l|}{ Gestational age } \\
\hline Full term & $26(100 \%)$ & $13(100 \%)$ & \multirow[t]{2}{*}{1} \\
\hline Preterm & $0(0 \%)$ & $0(0 \%)$ & \\
\hline \multicolumn{4}{|l|}{ Recent antibiotic use } \\
\hline Yes & $8(30.8 \%)$ & $4(30.8 \%)$ & \multirow[t]{2}{*}{1} \\
\hline No & $18(69.2 \%)$ & $9(69.2 \%)$ & \\
\hline
\end{tabular}

was associated with stool positivity (61.5\% in positive samples vs $23.4 \%$ in negative samples, $\mathrm{p}=0.006\left(\chi^{2}\right)$, table 1A). This study may underestimate prevalence rates as stool positivity may be lower than respiratory samples.

We successfully sequenced the SARS-CoV-2 genome from all positive samples (full methods in online supplemental data), with variant identification achieved for five samples (online supplemental figure 1B). We performed V4 16S rRNA gene sequencing on samples using DADA2 and the SILVA database for microbiome taxonomic profiling. We compared microbiomes using a 1:2 case-control match, controlled for ethnicity, age, delivery mode, gestational age, gender and recent antibiotic use (table 1B). Differential species abundance testing was performed using DESeq2 contrasting the SARS-CoV-2 positive and control samples. We found a significantly different relative abundance of taxa (adjusted $\mathrm{p}<0.05$ ) between positive and control samples (all significantly different taxa at a species level shown in figure 1). Notably, we found a decreased abundance of Bifidobacterium bifidum and Akkermansia muciniphila in positive samples, both of which are linked to protection against inflammation. ${ }^{8} 9$ Bifidobacterium are also pioneering colonisers of the gut microbiota and have immunomodulatory properties. ${ }^{10}$ Bifidobacterium bifidum was found to be inversely correlated with disease severity in adults. ${ }^{1}$ While Yeoh et al ${ }^{1}$ saw differences in beta diversity, our microbiome changes may be less robust compared with those symptomatic patients, with no differences seen in alpha or beta diversity. Detection of changes may also be limited by sample size.

We show that microbiome changes are detectable even in asymptomatic infants infected with SARS-CoV-2. Of relevance, there is a decrease in antiinflammatory taxa, similar to that seen in symptomatic adults. The impact of this on the developing microbiome, and subsequent immune and inflammatory responses is unknown, but deserves further exploration given the risk of development of autoimmune and autoinflammatory conditions in children with COVID-19.

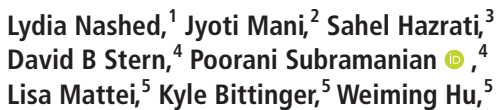
Lisa Mattei, ${ }^{5}$ Kyle Bittinger, ${ }^{5}$ Weiming Hu, ${ }^{5}$ 


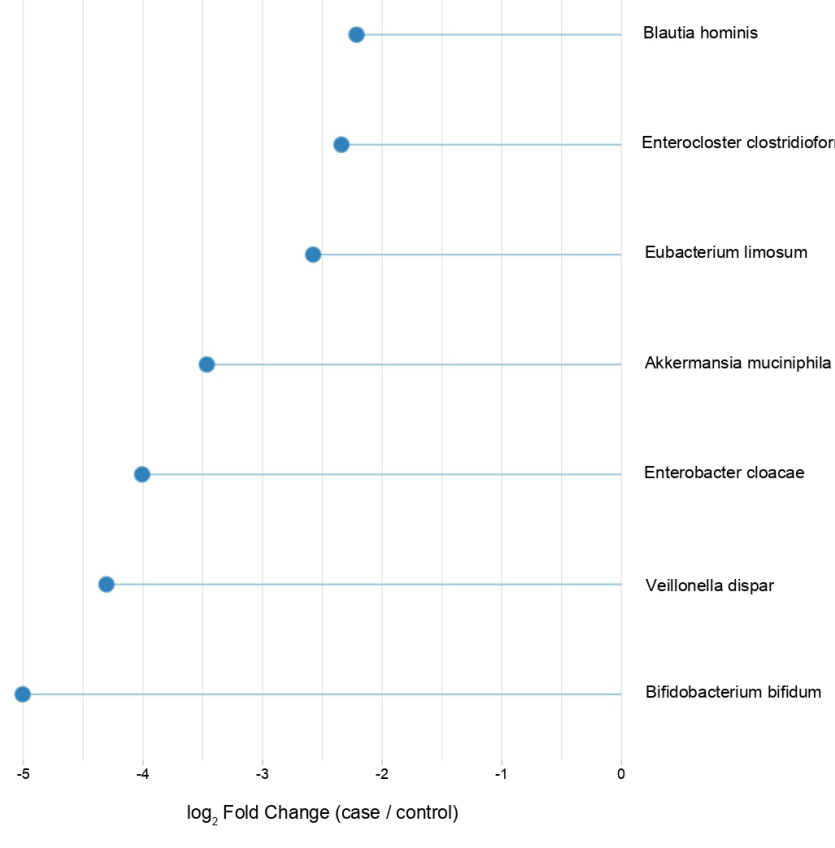

Species 